\title{
Physically Compatible Machine Learning Study on the Pt-Ni Nanoclusters
}

\author{
Huijie Zhen ${ }^{1 \#}$, Liang Liu ${ }^{12, \#}$, Zezhou lin, ${ }^{1}$ Siyan Gao ${ }^{1}$, Xi Zhang ${ }^{1 *}$ \\ ${ }^{1}$ Institute of Nanosurface Science and Engineering, Guangdong Provincial Key Laboratory of \\ Micro/Nano Optomechatronics Engineering, Shenzhen University, Shenzhen, 518060, China \\ ${ }^{2}$ Key Laboratory of Optoelectronic Devices and Systems of Ministry of Education and Guangdong \\ Province, College of Optoelectronic Engineering, Shenzhen University, Shenzhen, 518060, China
}

\#These authors contribute equally

\section{Corresponding Author}

*email: zh0005xi@,szu.edu.cn 
Table of contents

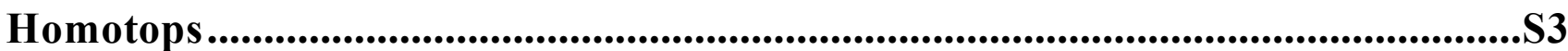

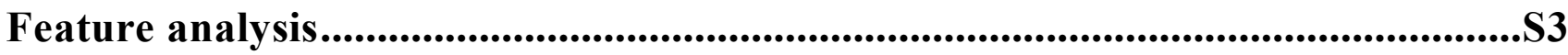

Crossover mode

Niche genetic algorithm search result .................................................................................55

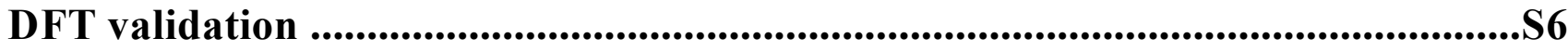

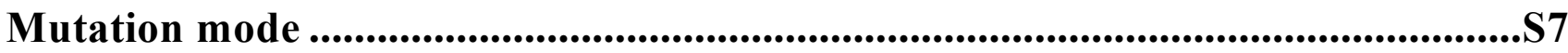




\section{Homotops}

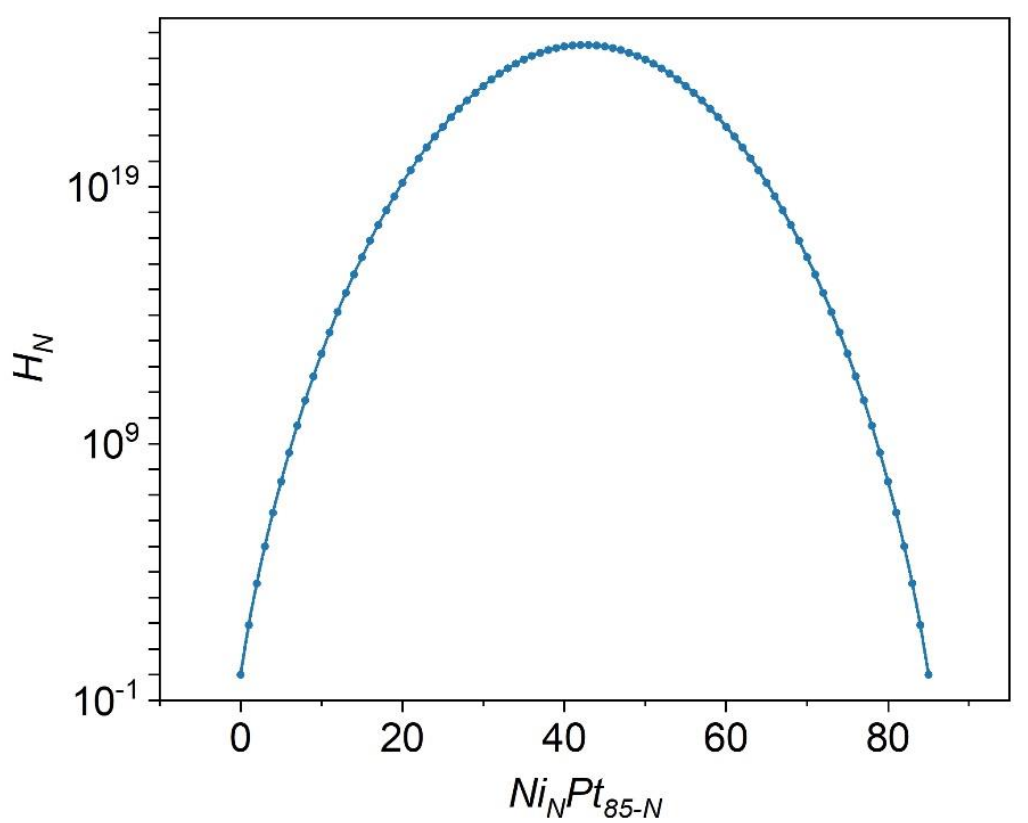

Fig. S1 The number of homotops varies with the composition of Pt-Ni alloy nanoclusters.

\section{Feature analysis}

As shown in Fig. S2a, the value of the segregation-extent BOP is proportional to the excess energy calculated by DFT. This means that strong Ni-Pt bonding leads to more stable Pt-Ni alloy nanocluster. The shell-resolved undercoordinated ratio also shows a positive correlation trend with the excess energy calculated by DFT, as shown in Fig. S2b. 
a

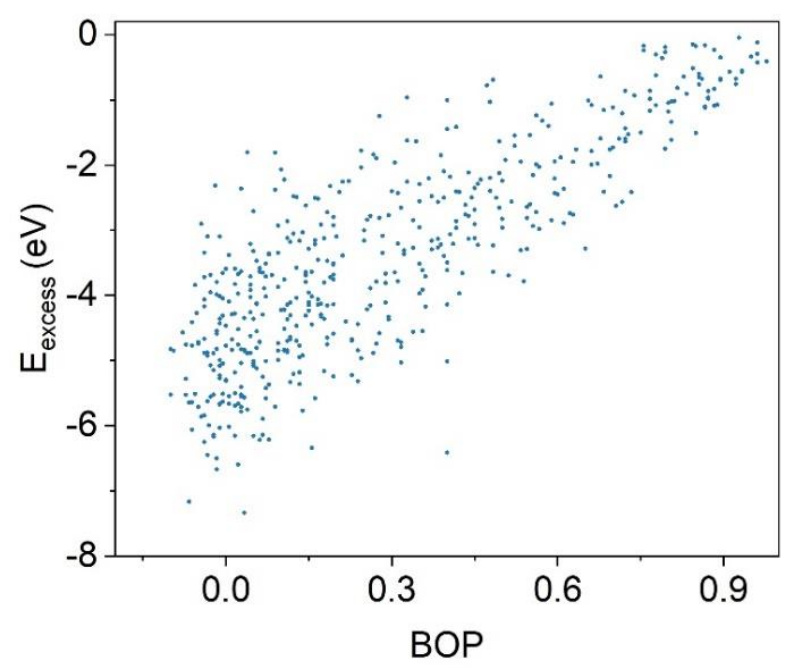

b

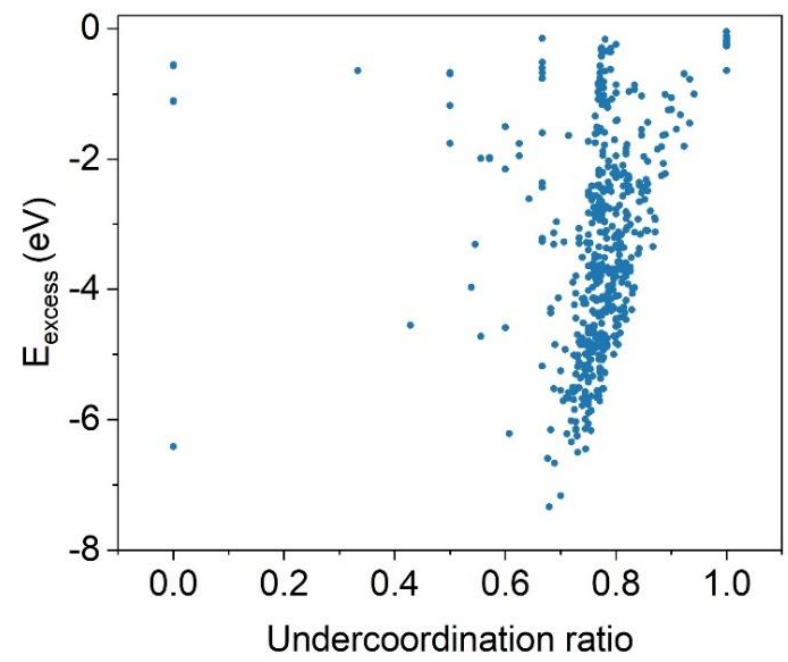

Fig. S2 a The influence trend of BOP on excess energy. b The influence trend of undercoordination ratio on excess energy.

\section{Crossover mode}

At the beginning, we generate the random number seed $1 \in[0,1]$. If seed 1 is less than the crossover probability $\mathrm{Pc}$, the crossover mode is set to crossover-inside; else, a random number seed $\epsilon \in$ $[0,1]$ is generated. If seed 2 is greater than the crossover probability Pc, the crossover mode is set to retain individuals; else, it is set to crossover-outside. 


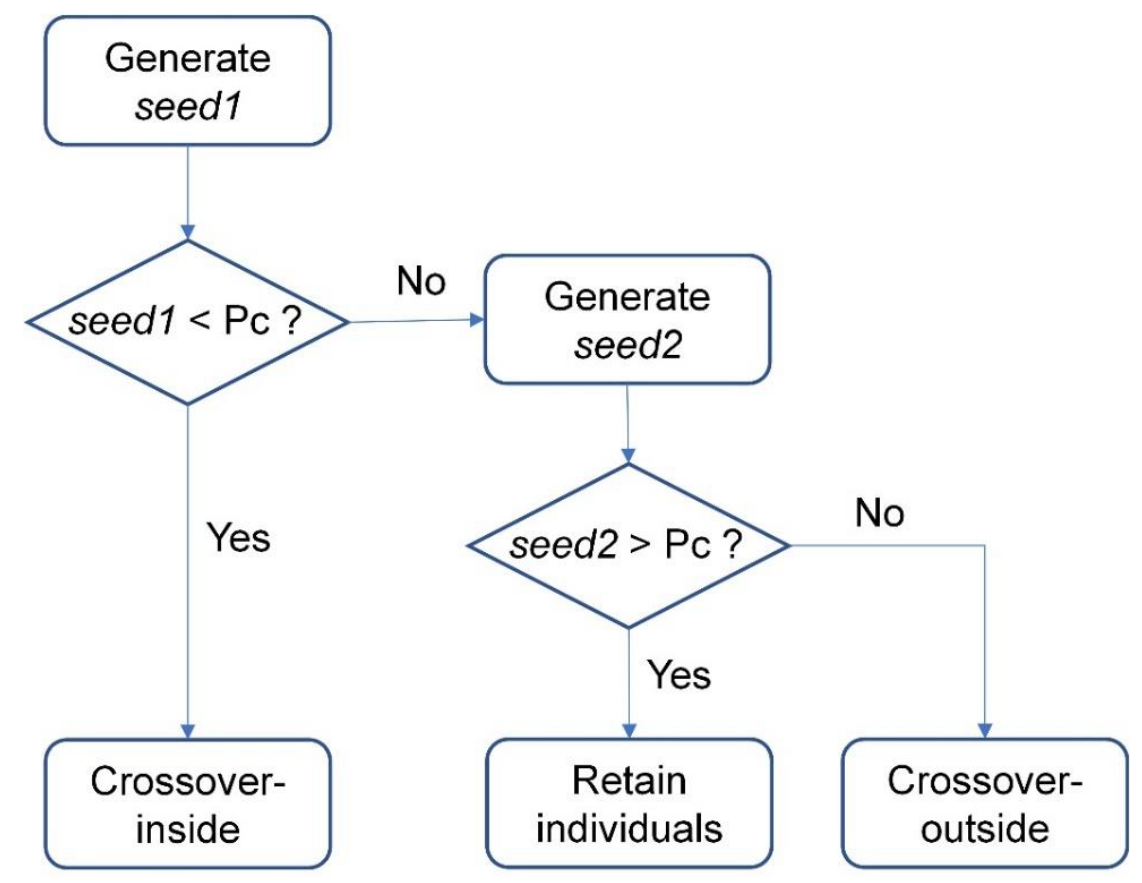

Fig. S3 The flowchart of crossover mode of niche genetic algorithm

\section{Niche genetic algorithm search result}

The physically niche genetic-machine learning model is used to search the $\operatorname{Pt}(85-\mathrm{x})-\mathrm{Ni}$ alloy nanoclusters for 5 times. The search results are shown in Fig. S4a, and the algorithm convergence curves are shown in Fig. S4b.

a

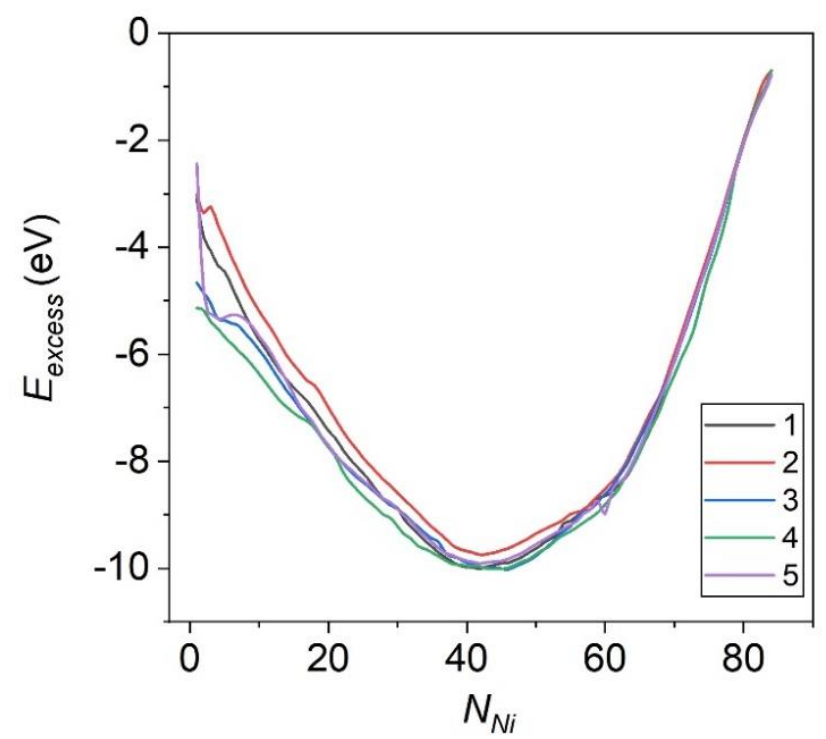

b

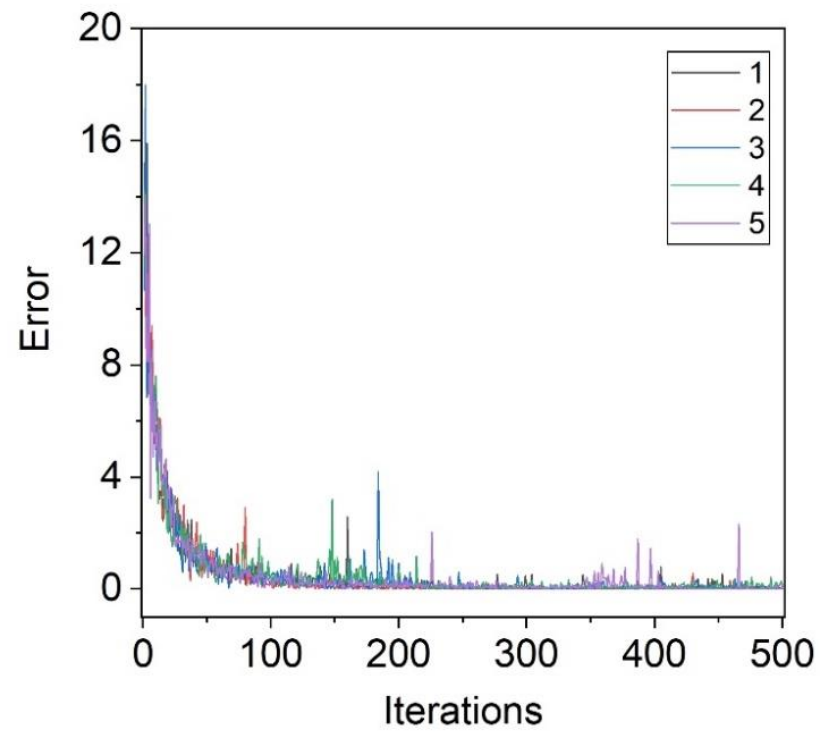


Fig. S4 a The search results of physically niche genetic algorithm combined with gaussian process regression (GPR). b The convergence profile for the physically niche genetic algorithm combined with GPR search. The error value is the cumulative of the difference in the excess energy of the optimal individual in each niche between two generations.

\section{DFT validation}

We have added the DFT calculation of $x=37 \sim 47$ in $\mathrm{Ni}_{\mathrm{x}} \mathrm{Pt} 85-\mathrm{x}$, and calculated the absolute percentage error of DFT calculation energy and ML potential energy, as shown in Figure R1. It can be seen that the error between the DFT calculation energy and the ML potential energy is relatively small, the prediction error of each component does not exceed $6 \%$, which fully proves the high accuracy of machine learning potential.

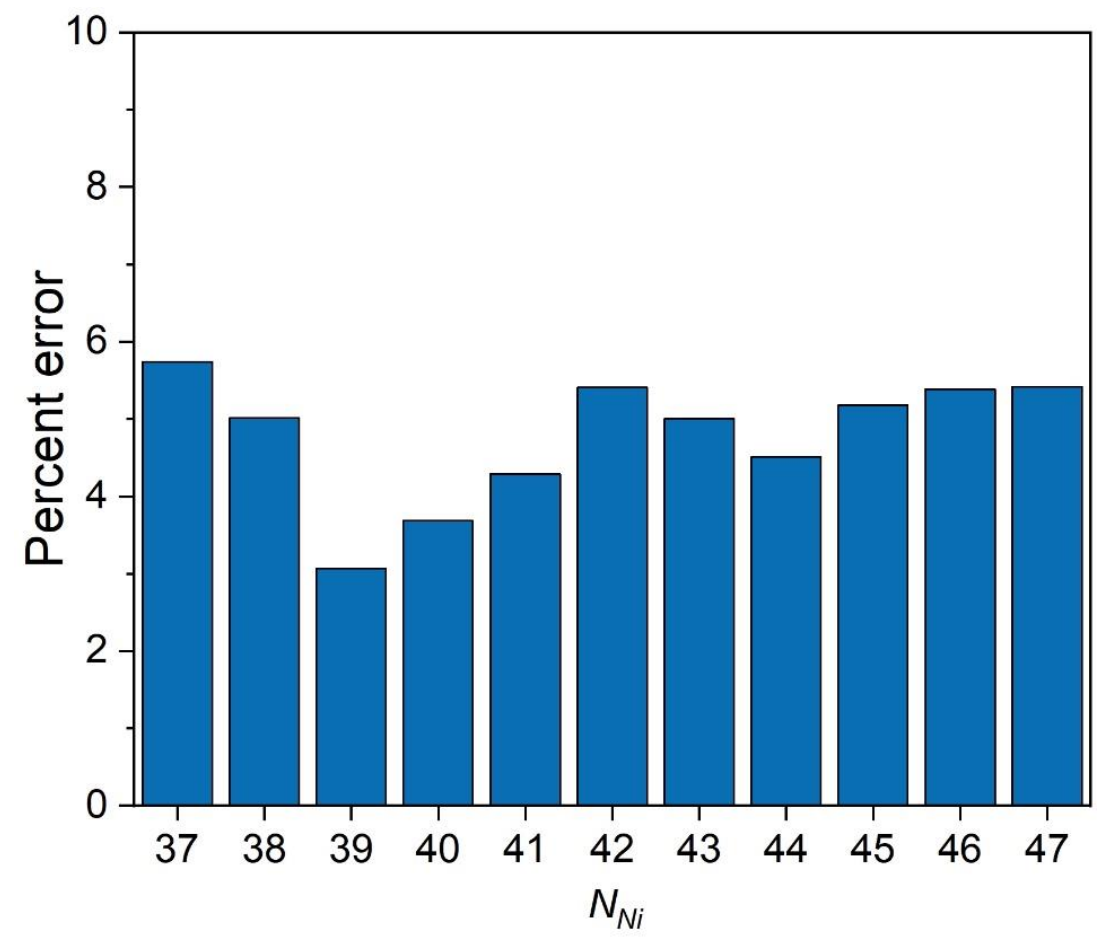

Fig. S5 For the structural region of $x=37 \sim 47$ in $\mathrm{Pt}(85-\mathrm{x}) \mathrm{Nix}$, the absolute percentage error of DFT calculation energy and machine learning potential energy. 


\section{Mutation mode}

The selection mechanism of mutation mode is shown in Fig. S6a, the exchange mutation and onsite mutation steps are serial. Figure S6b shows the implementation of the two types of mutation. Exchange mutation is to randomly exchange the positions of atoms of different element type, so as to explore the impact of atomic ordering on energy. Onsite mutation is to randomly select an atom and change its element type, thereby exploring the impact of alloy nanocluster composition on energy. Onsite mutation does change the niche to which the alloy nanoclusters belong, but in the process of the algorithm, we use the elite retention strategy to maintain the integrity of the niche. The elite retention strategy refers to the parent generation through genetic operation to produce offspring, and then select the best individuals in each niche from the offspring to compete with the parents in each niche, thereby replacing the low fitness individuals in the parent. Therefore, even if onsite mutation causes changes in the niche of alloy nanoclusters, the complete existence of 84 niches will not be destroyed.

a

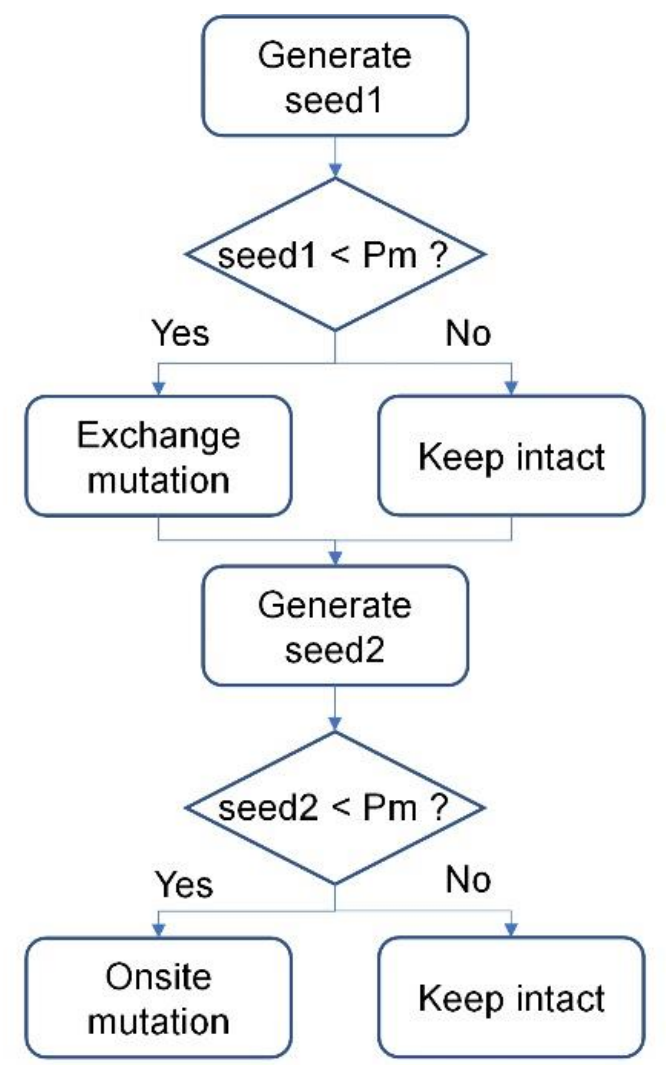

b

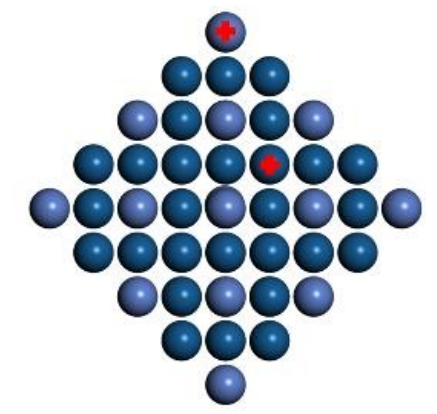

Exchange mutation

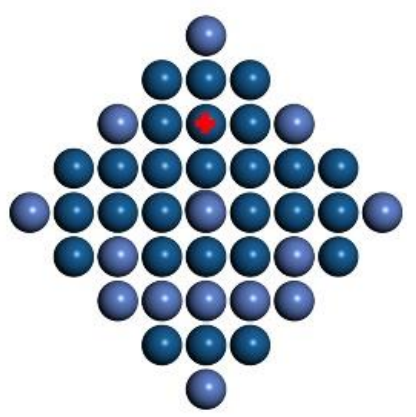

Onsite mutation

Fig. S6 a The flowchart of mutation mode of niche genetic algorithm. b Schematic of two mutation types. Blue and purple represent $\mathrm{Pt}$ atoms and $\mathrm{Ni}$ atoms, respectively. 